\title{
Políticas sociales y redes políticas. La implementación de un programa de economía social en un municipio de Argentina
}

\section{Social policies and political networks. The implementation of a social economy program in a municipality in Argentina}

doi: http://dx.doi.org/10.32870/espiral.v25i72.6526

Matías José lucci*

\begin{abstract}
Resumen
En este trabajo se analiza la implementación del programa social Argentina Trabaja en un municipio de la provincia de Buenos Aires, Argentina, con el objetivo de mostrar que las intenciones del programa (fomentar relaciones sociolaborales en el marco de la economía social) se vieron afectadas, en este municipio, por las características de las redes políticas con las que se implementó. A partir de este caso, se sostiene que, aunque por un lado las redes políticas permiten y agilizan el funcionamiento burocrático, ya que a través de ellas los programas sociales nacionales llegan hasta sus destinatarios, por el otro, limitan a los objetivos programáticos. Se utilizó un enfoque cualitativo que combinó observación participante y entrevistas a profundidad a funcionarios municipales $y$ destinatarios del programa.
\end{abstract}

Palabras clave: política social, redes políticas, Argentina Trabaja, burocracia estatal, implementación de políticas.

\begin{abstract}
This paper analyzes the implementation of the Argentina Trabaja social program in a municipality of Buenos Aires, Argentina, with the aim of showing that the intentions of the program to promote social and labor relations in the social economy were affected, in this municipality, by the characteristics of the political networks by which it was implemented. From this case, we argue on the one hand that political networks allow and accelerate the functioning of the State, since through them national social programs reach their recipients, but on the other hand, they limit the programmatic objectives. The work was done through a qualitative methodology that combined participant observation and in-depth interviews with municipal officials and recipients.
\end{abstract}

\footnotetext{
Keywords: Social policies, political networks, Argentina Trabaja, state bureaucracy, policies implementation.
}

\footnotetext{
- Investigador del Instituto de Investigaciones en Humanidades y Ciencias de la Educación de la Universidad Nacional de la Plata (UNLP-CONICET), Argentina. ORCID: http://orcid.org/00000002-6250-4277—matiasiu3@gmail.com

Fecha de recepción: 10 de febrero de 2017. Fecha de aceptación: 16 de febrero de 2018 .
} 
Introducción'

Varios programas sociales gubernamentales en Argentina entre 2003 y 2015 buscaban la inclusión social a partir de la generación de puestos de trabajo (Alonso y Di Costa, 2015), al tiempo que fomentaban relaciones sociolaborales entre sus destinatarios según principios y valores de la economía social.

Argentina Trabaja es uno de esos programas. Se trata de un programa que depende del Ministerio de Desarrollo Social de la Argentina, que comenzó a implementarse en 2009 y que propone integrar a sus destinatarios en cooperativas de trabajo bajo las consignas y valores del cooperativismo, la igualdad y la solidaridad (Iucci, 2010).

Este trabajo tiene como objetivo estudiar la implementación de dicho programa social en un municipio ${ }^{2}$ de la provincia de Buenos Aires, Argentina. Se entiende que analizar la implementación de políticas conociendo a los actores y relaciones en las que entran es un punto de partida interesante para caracterizar y dar cuenta de la intervención estatal y su funcionamiento cotidiano, tema general al que se propone aportar conocimiento a partir de este caso. ${ }^{3}$

Desde esta perspectiva, la aspiración es caracterizar las redes políticas activas entre los agentes que implementaron este programa en el municipio elegido. Las preguntas centrales del trabajo son: ¿qué características presentan las redes políticas en este municipio? y ¿cómo afectan a la concreción de objetivos ministeriales?

I. Una versión previa de este artículo se presentó en el Second Forum ISA of Sociology, 20I2, en Buenos Aires.

2. Los nombres de la ciudad y de las personas que se utilizan en el trabajo son ficticios. Más adelante, se hace referencia al municipio en que se trabajó como El Saladero. Con esto, se intenta preservar el anonimato de las personas y lugares involucrados. 3. Se siguen, en este sentido, las sugerencias de Oszlack y O'Donnell (1995) para encarar estudios sobre el Estado. 
Interesa mostrar cómo la implementación de este programa se vio afectada por las características de las redes previamente constituidas en el nivel municipal. Específicamente, se sostiene que, por un lado, estas redes permiten y garantizan el funcionamiento del Estado, ya que a través de ellas los programas sociales nacionales llegan hasta sus destinatarios, pero, por otro lado, afectan (limitando) a sus objetivos programáticos.

El trabajo se realizó a través de una metodología cualitativa que combinó observación participante en diversos ámbitos de implementación del programa en dicho municipio, tales como las oficinas municipales vinculadas a su gestión (Oficina de Cooperativas, Secretaría de Producción, y espacios de trabajo de las cooperativas conformadas), y entrevistas a profundidad realizadas durante el año 2010 a funcionarios encargados de su implementación y a destinatarios del programa. Se completó información con documentos ministeriales sobre el programa.

El artículo se organiza de la siguiente manera. Primero, se discuten algunas ideas sobre redes políticas e implementación de programas. Luego, se presentan rasgos generales del programa social en cuestión, y tras esto se brinda información sobre las características de las redes políticas previamente constituidas en el municipio analizado para la implementación del programa. Por último, se caracterizan las formas bajo las cuales esta modalidad de implementación adoptada afectó a los objetivos programáticos en el caso de estudio.

\section{Implementación de programas y redes políticas}

Como se ha mencionado, el presente trabajo pretende aportar conocimiento sobre el funcionamiento del Estado, principalmente respecto de la burocracia estatal, a partir del estudio y análisis de las políticas que implementa. 
En gran medida, los estudios sobre implementación de políticas se han mostrado preocupados por abordar su fracaso (Aguilar Villanueva, 1994). La principal búsqueda que ha orientado a estos estudios se concentra en torno a indagar por qué fallan las políticas. Es decir, si las políticas se encontraban meticulosamente elaboradas y contaban con los recursos necesarios para llevarlas adelante, ¿qué explica el hecho de que los objetivos no se cumplieran? (Pressman y Wildavsky, 1998).

El fracaso de las políticas se ha explicado con base en una combinación de dimensiones tales como la inflación de objetivos dispuestos en sus diseños, la desatención del personal burocrático sobre las orientaciones y sentidos de los lineamientos programáticos, las resistencias del personal administrativo a implementar tal o cual programa, los desencuentros, incluso los conflictos intergubernamentales con vistas a acordar estrategias comunes de gestión, la heterogeneidad de intereses entre actores involucrados, y la indiferencia de los destinatarios de las políticas. Con ello, estos estudios generaron ya un conocimiento sobre las dificultades de concertar la acción conjunta y coordinada según los objetivos programáticos elaborados de todos los actores intervinientes.

Otros trabajos, escritos en las décadas de los setenta y los ochenta, atendieron el rol de los administradores vinculados directamente con la atención directa al público para explicar el resultado de tal o cual política (Lipsky, 1980). Nuevamente, guiados por la preocupación del fracaso de las políticas, los analistas comenzaron a destacar dimensiones tales como la relación entre las responsabilidades asumidas y la capacidad para cumplirlas, o el desempeño de los actores de ventanilla, caracterizando con ello a la evasión de tareas y las resistencias a programas, elementos que ayudaban a dar cuenta de las dificultades para la concreción del éxito de las políticas. 
Se entiende que los trabajos sobre implementación de políticas realizaron un aporte sustancial al conocimiento sobre la burocracia estatal, ayudando a caracterizarla como una organización compleja, compuesta por múltiples agencias que operan en distintos niveles estatales, aunque no necesariamente en una misma dirección, y cuyos trabajadores mantienen relaciones, no siempre fáciles, a propósito de las políticas. Se destaca que ello ha sido relevante a la hora de desacralizar la imagen de una burocracia estatal ágil, homogénea, eficiente, racional, tal como se desprendía del tipo ideal weberiano, y avanzar en una agenda de investigación sobre las realidades de las burocracias estatales.

La investigación sobre implementación de políticas sociales en el nivel municipal ha sido influenciada por estas perspectivas señaladas. Es decir, se ha tendido a indicar principalmente las dificultades y los obstáculos de la burocracia local para llevar adelante los programas nacionales.

En términos generales, se argumenta que las capacidades estatales y municipales no se encuentran bien provistas para afrontar responsabilidades tales como la implementación de programas sociales complejos (Isuani, 2009). Dentro de esta argumentación general, se ha prestado especial atención a las dificultades implicadas en coordinar acciones entre agencias estatales de distinto nivel para implementar políticas (Isuani, 2009; Alonso, 2010). También se ha colocado el foco en las dificultades existentes para concertar la cooperación y el trabajo en conjunto entre dependencias administrativas del mismo nivel gubernamental (Ilari, 2002a y 2002b; Cravacuore, Ilari y Villar, 2004; Cravacuore e Israel, 2007).

Chiara y Di Virgilio (2005), buscando explicar por qué la implementación de políticas no responde siempre a su formulación original en el nivel municipal, argumentaron, en discusión con la tradición clásica recién mencionada, que existen elementos presentes en aquello que denominaron el régimen local de implementación que actúan de 
modo tal que permiten mitigar los defectos vinculados a las debilidades de las capacidades estatales instaladas. A partir de su caso de estudio, mostraron que los programas que llegaban al municipio eran resignificados de acuerdo a un entramado de intereses y pretensiones de cada uno de los actores involucrados. Consecuentemente, los programas sociales nacionales se implementaban, pero de un modo diferente a lo que se proponía en la planificación nacional.

La incidencia del clientelismo ha tenido también centralidad en estos estudios, principalmente aquella que se ha denominado de tipo instrumental, ${ }^{4}$ denominada así en tanto los patrones de tipo instrumental pondrían en circulación, estratégicamente, planes y programas sociales con base en un cálculo establecido para obtener réditos políticos. En esta línea de argumentación, el Estado pondría en circulación planes sociales entre los destinatarios a través de mediadores -patrones de redes clientelares-, y estos otorgarían lealtades políticas expresadas, por ejemplo, en asistencia a actos políticos y, eventualmente, en el voto en contexto electoral (Torres, 2002; Di Natale, 2004).

Cabe mencionar que otras visiones (Durston, 2005) se abren hacia otras concepciones sobre clientelismo y matizan esas conclusiones, mostrando que los intermediarios de esas redes no necesariamente mantienen la única motivación vinculada al crecimiento individual a través de búsqueda de clientelas, sino que en su accionar también desarrollan compromisos no personales, se comprometen con sus roles, y actúan en función de una noción de bien común y desarrollo comunitario. Y en este sentido, pueden favorecer a la producción y redistribución de bienes públicos (Ortiz de Rozas, 2017).

Teniendo en cuenta estos estudios previos, se propone colocar el foco de este trabajo en las redes políticas activas

4. Existe una gran controversia acerca del significado, extensión y capacidad explicativa del clientelismo.Aquí, se utiliza esta denominación ideada porVommaro y Quirós (20II) para precisar el tipo de concepción con la que se está discutiendo. 
en un municipio para la implementación de programas sociales que encuentran su origen en el nivel nacional.

La idea de redes políticas que se presenta en este trabajo se nutre principalmente de dos fuentes bibliográficas: por un lado, de los estudios y los debates sobre el lugar y la extensión del clientelismo en la implementación de políticas sociales, principalmente, aunque no exclusivamente, en Argentina (Auyero, 1997 y 2001; Durston, 2005; Soprano, 2008; Vommaro y Quirós, 2011; Zarazaga, 2015), y, por el otro, de la noción de redes de política pública o policy networks, orientada a pensar la acción horizontalmente arreglada entre actores e instituciones públicos y privados que facilitan la implementación de programas (Jordana, 1995; Chaqués Bonafont, 2004; Bebbington, 2005; Klijn, 2005; Zubriggen, 2011).

El concepto de red política puede ser útil para observar el modo en que durante la implementación de la política se combinan prácticas y relaciones entre actores que poseen distintos anclajes y vínculos con agencias burocráticas en función de los diversos niveles de gestión involucrados, que poseen múltiples identidades conformadas por sus involucramientos en ámbitos de sociabilidad (partidarias, familiares, barriales, de amistad, de empleos anteriores, etc.), y que también presentan urgencias, intereses y motivaciones diversos en torno a los programas que envía el Estado nacional, pero asumen un grado de compromiso con la política pública.

Se resalta en este trabajo especialmente el hecho de que la red por la que se implementa el programa se encuentra preconstituida, es decir, que tiene una historia: en el municipio analizado, había una trama social y política anterior a la llegada de los programas nacionales y una modalidad de trabajar con programas sociales provinciales y nacionales por parte de los actores municipales que antecedían al nuevo 
programa enviado por el ministerio y que condicionaron su desempeño.

\section{El programa Argentina Trabaja: inclusión social en el marco de la economía social}

Este trabajo constituye un estudio de caso (Yin, 2013) realizado en el marco de la metodología de investigación cualitativa, que combinó la observación participante en actos y eventos vinculados con la implementación de programas sociales en este municipio con entrevistas semiestructuradas y a profundidad y charlas informales con actores encargados de su implementación entre 2007 y 2010. Se complementó la investigación con información proveniente de documentos programáticos y publicaciones del Ministerio de Desarrollo Social de Nación y del municipio sobre el programa.

El trabajo de campo se realizó en una ciudad de la provincia de Buenos Aires de alrededor de 100000 habitantes que se denomina, para los fines de este trabajo, como El Saladero. Este municipio sufrió particularmente la desindustrialización, consecuencia de reformas económicas de corte neoliberal implantadas fuertemente en la década de los noventa. Los índices de pobreza y desempleo en la localidad fueron en aumento desde entonces, como también lo hizo la cantidad de destinatarios de programas sociales. La reindustrialización y la creación de puestos de trabajo fueron temas instalados en la agenda municipal y preocupación central de las autoridades municipales desde ese momento. En este contexto, la llegada del programa Argentina Trabaja tuvo una importancia central para las autoridades.

El Programa Ingreso Social con Trabajo Argentina Trabaja (o simplemente Argentina Trabaja) se creó en 2009 a través de una resolución del Ministerio de Desarrollo Social de la Nación, y se proyectaba como aplicable a todo el territorio 
nacional. Entre los objetivos del programa se encontraban: "la promoción del desarrollo económico y la inclusión social a través de la generación de nuevos puestos de trabajo genuino, con igualdad de oportunidades, fundado en el trabajo organizado y comunitario incentivando e impulsando la formación de organizaciones sociales de trabajadores" (Ministerio de Desarrollo Social de la Nación, 2009, p. 10).

Esto significaba que, por un lado, se intentaba acercar a la población al mercado laboral, y, por el otro, incorporar a sus destinatarios en cooperativas de trabajo o de servicios aspirando a promover valores solidarios y cooperativos entre ellos.

En cuanto a la población destinataria, se afirmaba que "Está destinado a personas sin ingresos formales en el grupo familiar, ni prestaciones de pensiones, jubilaciones nacionales ni otros planes sociales, a excepción del Programa de Seguridad Alimentaria" (Ministerio de Desarrollo Social de la Nación, 2010, p. 6), con lo cual puede ser entendido como un programa que mantenía un criterio de focalización sobre una franja poblacional como criterio de selección de sus destinatarios.

La implementación del programa podía ocurrir a través de dos vías alternativas: la burocracia municipal o los movimientos sociales, aunque bajo supervisión del Estado. Según fuentes oficiales, en junio de 2011 se habían creado en todo el país 2022 cooperativas que involucraban a alrededor de 190000 cooperativistas (Ministerio de Desarrollo Social de la Nación, 2011). Cada cooperativa se conformaba por aproximadamente sesenta personas y tenía una cúpula dirigente (presidente, tesorero, síndico, vocales y socios), que debía tomar las decisiones más importantes en asambleas, de las que participaban todos sus socios.

Las cooperativas conformadas en el marco de este programa eran contratadas por el municipio para la realización de distintas obras. Individualmente, cada cooperativista 
recibía un incentivo de inclusión social de alrededor de ARG 1200.00 mensuales (es decir, cerca de USD 300.00 de acuerdo al tipo de cambio del momento), un monotributo social, y con ello, el acceso a una obra social y un seguro por accidentes de trabajo.

El programa tenía algunos antecedes relevantes en otros planes y programas de empleo: en Argentina fueron centrales el denominado Plan Trabajar, hacia fines de la década de los noventa, y su sucesor, el Plan Jefas y Jefes de Hogar Desempleados, pensado principalmente para paliar el desempleo dejado por las políticas de la década de los noventa y la coyuntura crítica del 2001. Años más tarde, y con el kirchnerismo, se diseñó el plan Manos a la Obra, con el que los programas sociales del Ministerio de Desarrollo Social de la Nación se comenzaron a enmarcar dentro de la economía social. Justamente en ese programa existía un lineamiento específico vinculado a la creación de cooperativas de trabajo, que es un antecedente directo a Argentina Trabaja.

Argentina Trabaja apuntaba en el largo plazo a emancipar a las cooperativas conformadas respecto de la contratación estatal e incentivar su participación en el mercado laboral en tanto cooperativas.

Entre los principales logros del programa presentados por el ministerio se encontraban: la mejora sensible de los ingresos de los hogares con destinatarios del programa, la extensión de la capacitación en un oficio para sus destinatarios, la incorporación laboral para jóvenes sin experiencia, la extensión del sistema de protección social (a través de una obra social), y la inclusión en el sistema financiero a través de su bancarización (Ministerio de Desarrollo Social, 2011). 
A continuación, se presenta cómo se implementó el programa en El Saladero. Así, las áreas municipales involucradas en la implementación del programa eran las siguientes: ${ }^{5}$

- Secretaría de Obras Públicas.

- Oficina de Cooperativas (del centro).

- Oficina de Cooperativas (zona alejada).

- Corralón o depósito municipal.

- Coordinadores barriales.

En el armado y planificación del programa en el nivel municipal, se encontraban fundamentalmente involucradas la Secretaría de Obras Públicas, la Secretaría de Producción Municipal y la de Promoción Social. ${ }^{6}$ A continuación, se describen las tareas principales que encaraban cada una de ellas.

En la Secretaría de Obras Públicas, se proyectaban las obras que las cooperativas iban a ejecutar en la ciudad, y también se atendían el aspecto contractual entre las partes (municipio y cooperativa) y la supervisión sobre el desarrollo y finalización de las obras realizadas.

Dependiente de esta secretaría y de la Oficina de Empleo Municipal estaba la Oficina de Cooperativas. Esta tenía las principales actividades y responsabilidades. En esta oficina, se encargaban de la conformación de cooperativas, de la organización y reglamentación sobre las ausencias a la jornada laboral, y de atender y responder a las solicitudes hechas por los agentes ministeriales sobre el desarrollo del programa.

La Oficina de Cooperativas tenía también otro sitio de funcionamiento en el centro de la ciudad. En esa oficina se inscribían todos los aspirantes del programa, se les hacía una entrevista para dejar constancia de su trayectoria labo-

5. Según información obtenida a través de entrevistas personales (20 l la, 20 I lc, 20 I I y 20 Iff y de observación participante en la mencionada dependencia. 6. En el organigrama municipal, la secretaría tenía el carácter de jerarquía. De ella dependían las direcciones, $y$ de estas, a su vez, las oficinas. Además de estas tres secretarías, se encontraban en el municipio dos más: Gobierno y Economía. Las cinco secretarías conformaban el conjunto del Poder Ejecutivo municipal. 
ral, y esta información era luego enviada a la otra oficina de cooperativas, donde seleccionaban a los destinatarios del programa.

En el corralón municipal, se encargaban de realizar las compras de los materiales que requerían las cooperativas en su trabajo y de redistribuirlos entre los distintos lugares de trabajo donde estaban las cooperativas.

Por último, los coordinadores barriales estaban encargados de tomar asistencia de destinatarios a la jornada, de supervisar el trabajo cotidiano de las cooperativas, y de solucionar los conflictos frecuentes que ocurrían entre estos.

Por otra parte, en la Secretaría de Promoción Social se encargaban principalmente de tramitar los seguros de trabajo, el monotributo social, así como otros beneficios que tenían los destinatarios de este programa.

\section{La implementación del programa}

Con el fin de analizar la implementación del programa, se retoma a Repetto (2005), quien afirmó que en el momento de la implementación de políticas sociales se toma un conjunto de decisiones que tienen una naturaleza decididamente política, ya que se define cómo se va a llevar adelante tal o cual programa. La pregunta por el cómo implica, para la perspectiva de este trabajo, decisiones que refieren a con quiénes, es decir, a cuáles actores se van a contactar para implementar el programa, y también a para quiénes, o, en otras palabras, a qué actores se iban a destinar los programas.

¿Con quiénes? y ¿para quiénes? se vuelven, a su vez, preguntas que nos depositan en el centro de las redes políticas en las que se encontraban tanto quienes implementaban los programas como sus futuros destinatarios. La exposición siguiente se realiza bajo estas dos preguntas sustanciales: ¿con quiénes? y ¿para quiénes? 
En las páginas siguientes, se argumenta que en los siguientes programas estas preguntas pueden ser respondidas vinculándolas con los entramados sociopolíticos preexistentes a la llegada del programa al municipio.

3. I. ¿Con quiénes se implementó el programa?: redes político-partidarias y el Gobierno municipal

Interesa rastrear, además de las principales actividades de cada una de las dependencias administrativas, los principales perfiles sociopolíticos de estos directivos. ¿Quiénes estaban al frente de estas dependencias? Ello es congruente con las intenciones del trabajo de rastrear las redes políticas y de mirar quiénes, como se desglosará en el próximo apartado, tenían incidencia en los resultados de la implementación.

En El Saladero, los principales funcionarios municipales de las secretarías, las direcciones y las oficinas estaban en relación con líneas y facciones del partido justicialista, históricamente gobernante en este municipio. ${ }^{7} \mathrm{La}$ ocupación de los principales puestos en la administración local se realizaba a partir de un intercambio establecido entre las principales agrupaciones políticas que pertenecían al partido justicialista local en un momento de campaña electoral. ${ }^{8}$ De este modo, el reverso de la estructura del organigrama municipal era un mapa de las facciones partidarias y del entramado de alianzas que daban lugar al triunfo electoral.

En el 2007, año electoral, cinco facciones en el peronismo local confluyeron en una alianza electoral con vistas al triunfo en las elecciones de aquel año. Tal como confirman los entrevistados para el presente estudio, quienes integra-

7. La parte que sigue se estructuró con base en información extraída a partir de entrevistas en profundidad con los secretarios, directores y el coordinador barrial mencionados en este apartado.

8. Tal como se desprendió de una entrevista realizada a uno de los líderes de una facción política aliada a la del intendente en aquella coyuntura. 
ron los puestos más importantes del Gobierno municipal a partir del 2007 eran líderes de esas facciones. ${ }^{9}$

Con respecto a las oficinas involucradas en la gestión de este programa, se puede destacar que el secretario de Obras Públicas era un líder de una de las facciones vinculadas a un barrio alejado de la ciudad que entró en alianza con la agrupación del intendente en el 2003 con vistas al triunfo electoral en las elecciones de ese año. El director de la oficina del centro pertenecía a una agrupación de otro barrio de la ciudad, también aliada a la agrupación del intendente desde 2003. En el centro, estaban dos hermanos que habían ingresado recientemente a la gestión, pero que habían trabajado en política con el intendente desde su ascenso político en el 2003.

Por su parte, Ramón, uno de los coordinadores barriales, era el encargado de las llaves que abrían la unidad básica ${ }^{10}$ de la agrupación donde militaba el intendente, y era también presidente de una cooperativa del programa.

A su vez, no todas estas oficinas municipales trabajaban articuladamente y coordinaban fácilmente la gestión de programas. Durante todo el 2008, era sabido por todos los integrantes del Poder Ejecutivo municipal que había una tensión entre el secretario de Obras Públicas y el de Promoción Social, desacuerdo que le costó el puesto a este último. También se presentó una tensión entre la reemplazante de dicho secretario y los coordinadores de la nueva oficina de cooperativas, creada a propósito de la llegada de este programa al municipio, en 2009.

9. Es una característica de los grupos que hacen política en este municipio que cada individuo que se desempeña en este espacio o bien conoce a los otros en términos personales o bien es capaz de referenciar su militancia o pertenencia a determinado sector político. Del mismo modo, quienes ocupaban cargos en el Gobierno municipal solían asociar tal o cual dependencia administrativa con el sector político de militancia. Algunos de ellos son familiares de otros militantes que se desempeñan en la política local, y muchos de ellos militantes históricos relacionados con el forjamiento del peronismo en la ciudad.

10. Unidad básica es la denominación de los locales partidarios del Partido Justicialista (Levitsky, 2005). 
Con estos elementos, puede pensarse que había un contacto previamente establecido entre estos actores a la llegada del programa, y que era posible rastrearlo al menos hasta la campaña electoral. A su vez, es posible pensar que en los momentos de gobierno, es decir, de gestión de políticas, estos actores continuaban en contacto, y como ya se señaló, que esas relaciones no siempre eran armoniosas.

Esto quiere decir que las redes políticas que en un momento tienen como sentido ganar elecciones eran útiles a la implementación de políticas en momentos de gobierno.

A continuación, se muestra que estos contactos incidían en las decisiones que tomaban estos actores con relación al programa. ¿Cómo se conformaban las cooperativas? y ¿quiénes ingresaban a las mismas? son preguntas que se agrupan en el para quiénes de la implementación.

\section{2. ¿Para quiénes se implementó el programa?:} entre los lineamientos programáticos y las redes político-administrativas

Si bien la letra escrita del programa afirmaba que quienes iban a recibir su apoyo eran individuos que cumplían ciertas características (personas sin ingresos formales en el grupo familiar ni prestaciones de pensiones, jubilaciones nacionales ni otros planes sociales, a excepción del Programa de Seguridad Alimentaria), la aplicación o no de estos criterios explicitados por los lineamientos programáticos era decisión de quienes implementaban el programa en el municipio.

$\mathrm{Al}$ momento de realización del trabajo de campo para el presente estudio, había cuatrocientos veinticuatro cooperativistas distribuidos en las cooperativas conformadas, mientras que el listado para ingresar al mismo superaba los 2000 inscritos. ${ }^{11}$ Es decir, era mucho mayor el número

I I. En 2010 y en 201 I, el programa tuvo más ingresos, sin embargo, el número de aspirantes era mayor que el de los que participaban. Esta información se obtuvo a través de entrevistas con funcionarios (20I l c y 20 I Id), que están en poder del autor del presente trabajo. 
de aspirantes que el de destinatarios efectivos. ¿Cómo decidían en la oficina de cooperativas municipal a quiénes incluir en el programa?

En aquel momento, el empleado de la oficina comentó sin rodeos: "Es clientelar. Entran de acuerdo a si tienen una participación política" (entrevista personal, 2011a). Los destinatarios del programa compartían la opinión de que poseer un contacto que los acercara a las oficinas municipales o pudiera interceder a su favor era necesario para ingresar a programa. "Entran sus familiares" (entrevista personal, $2011 b)$, comentó un destinatario del programa con tono de indignación en una de las entrevistas realizadas para el presente trabajo.

Lo dicho es una interpretación probable, si se entiende también que los líderes o referentes importantes de facciones políticas en el nivel local contaban, a su vez, con un número de clientes, es decir, personas a quienes se les prometía algún puesto burocrático o una ayuda para ingresar a un programa social en el caso de obtener el triunfo electoral. Bien podría resultar, tal como sugiere la bibliografía clásica sobre clientelismo de corte instrumental, que los votos se intercambian por favores.

Así, haciendo una digresión, por un lado, se entiende que la implementación de los programas requería de un criterio de decisión tomado por el equipo de trabajo de las oficinas municipales que ayudara a discriminar entre los aspirantes, y, también, que en este criterio primaban los contactos previos establecidos entre los actores: era más sencillo el ingreso al programa para quienes estaban vinculados a redes políticas que para quienes no lo estaban. ${ }^{12}$ No se pretende explicar el

12. A propósito de ello, el equipo realizador de este trabajo presenció un diálogo, ocurrido durante la etapa de campo, entre dos personas que eran destinatarias de otro programa social, pero que aspiraban a ingresar a Argentina Trabaja. Las dos se habían inscrito en la misma época en los listados de las oficinas municipales, pero mientras que una de ellas (identificada como Constanza) había logrado ingresar al programa, la otra (identificada como Carla) no. Constanza podía explicar por 
total de los ingresos de los destinatarios al programa, pero sí al menos afirmar que tener una relación establecida con mediadores de redes políticas era considerado por parte de los destinatarios como una ayuda para acceder a programas.

Por otro lado, y como un argumento que contrarresta esa interpretación de los actores intervinientes, y también de la bibliografía dominante en Argentina, en el ingreso de los destinatarios a este programa tiene carácter de explicación otro tipo de red política, una red que presenta un carácter más administrativo en tanto tiene la finalidad de facilitar la implementación de programas. Si bien esta red se complementa y se superpone con la red político-partidaria, puede afirmarse que no necesariamente tiene el carácter de clientelar instrumental que sugerían tanto los actores involucrados como la bibliografía predominante.

Si se revisa la modalidad bajo la que los integrantes de una de las cooperativas fueron llegando al programa, se encuentra que en muchos casos se trataba de destinatarios de programas de larga data, es más, muchos ya llevaban en aquel momento una trayectoria dentro de programas sociales de distinto tipo que era posible rastrear hasta el programa Jefas y Jefes de Hogar, que como se mencionó en apartados anteriores, formaba parte de los programas antecesores a Argentina Trabaja (entrevista personal, 2011e).

Como se mencionó, el programa Argentina Trabaja sustituyó en El Saladero al programa anterior, el plan Jefas y Jefes de Hogar, razón por la cual muchos de los que ingre-

qué había ingresado al programa Argentina Trabaja, y al mismo tiempo por qué su compañera no. La explicación se centraba en la relación más estrecha que tenía Constanza con mediadores políticos que se encontraban en el barrio donde vivía. Constanza venía incentivando desde hacía años esta relación, acompañando al referente político a múltiples actos partidarios. Carla, por el contrario, acababa de mudarse a la ciudad, aún no conocía a sus vecinos ni a quiénes podían ofrecerle este tipo de ayudas. Esta fue información extraída de charlas informales y observación participante en el marco de implementación del programa. 
saron a este programa pasaron directamente al nuevo una vez que comenzó su implementación.

Además, con el plan Jefas y Jefes de Hogar, ya se había formado un conjunto de contactos entre las oficinas municipales, coordinadores barriales y destinatarios de programas, es decir, había una red establecida entre estos actores que, al conectarlos, permitía el funcionamiento cotidiano de ese programa.

En el caso de varios de los integrantes de una cooperativa que con el programa Argentina Trabaja pasó a denominarse Mataco, la transición entre programas se originó de este modo: ellos trabajaban desde el año 2004 como destinatarios del plan Jefas y Jefes de Hogar, y se integraron a una cooperativa en el año 2009 en el marco del nuevo programa, tras el reclutamiento de su coordinador. De este modo, pasaron de un programa a otro. Para ellos, las actividades desarrolladas en uno y otro programa no habían cambiado tanto. Por el contrario, percibían una continuidad de tareas y actividades. ${ }^{13}$

Es importante destacar que los lineamientos programáticos de un programa y otro diferían sustancialmente en un punto: el programa anterior, Jefas y Jefes de Hogar, no incorporaba la idea de cooperativa y no estaba enmarcado dentro de la noción de economía social, sino que dicho programa reunía a un conjunto de personas bajo la supervisión de un coordinador, y estas realizaban actividades en forma conjunta en algún punto de la ciudad que eran dictaminadas también por la Secretaría de Obras Públicas municipal.

El hecho de que no percibieran la diferencia de las actividades que implicaba un programa y otro habla de que los lineamientos programáticos de este nuevo programa,

13. Esto no significa que el cambio se diera sin conflicto:estos viejos destinatarios del programa se incorporaron en una estructura nominalmente llamada cooperativa en la que se encontraron con otros integrantes, elementos que llevaron a enfrentamientos en el interior de la cooperativa entre viejos y nuevos cooperativistas. 
es decir, los valores propios de la economía solidaria que propusiera el ministerio nacional no se desarrollaban. Y puede entenderse que ello era así, en parte, porque la implementación del programa se vio afectada por la forma en que se encausaron programas sociales que ya tenían su mecánica establecida en este municipio, una mecánica que no involucraba la idea de cooperativismo.

\section{Conclusiones}

El presente trabajo ha pretendido aportar conocimiento al funcionamiento de la burocracia estatal, a partir del estudio y análisis de las redes políticas a través de las que se implementan políticas sociales. Para ello, se ha tomado el caso de la implementación del programa Argentina Trabaja en el municipio de El Saladero, en la provincia de Buenos Aires, en Argentina. Dicho programa fue presentado como novedoso por parte del ministerio nacional responsable, pues pretendía impulsar el desarrollo de las cooperativas en el marco de la economía social.

El presente trabajo comparte con gran parte de la bibliografía sobre implementación de políticas de vertiente clásica la idea de que los programas sociales que se presentan como novedosos desde la gestión nacional desvían sus objetivos en el nivel municipal. Considerando este caso, puede agregarse ahora que lo hacen porque en el nivel municipal hay una trama históricamente conformada antes de la llegada de los nuevos programas sociales que posee una dinámica y funcionamiento arraigado, y que en el caso de estudio aquí presentado, condicionó el rumbo del programa.

En el argumento que se detalló aquí, los actores que implementan programas nacionales en el nivel municipal se sitúan en dos tipos de redes que actúan de manera conjunta y simultánea, y que enmarcan su accionar: por un lado, una de naturaleza más política, vinculada a las redes partida- 
rias que pueden rastrearse hasta el contexto electoral y que continúan vigentes al momento de implementar programas, y por el otro, una más administrativa, que vincula a los funcionarios, coordinadores barriales y destinatarios desde programas anteriores. Las dos redes se complementan y se funden para dar lugar a la implementación del programa. Con la identificación de estas redes, se destaca la necesidad de avanzar hacia enfoques integrados de redes políticas que dialoguen y sitúen visiones que parecen disociadas, y que por sí mismas no podrían describir cabalmente el fenómeno.

Con respecto a las mencionadas redes de política pública, el presente estudio coincide con parte de la bibliografía respecto a la centralidad que tiene el análisis de las relaciones de horizontalidad que se presentan entre actores que implementan. Además, resulta central la concepción sobre que los actores participan y se muestran interesados en la gestión y en poner en marcha los objetivos de los programas, aunque también se entiende que estos tienden a concentrarse en aspectos más que nada institucionales, vinculados a reglas de juego formalmente escritas y sancionadas, y vigentes en un marco legal.

De este modo, se deja de lado la informalidad que se presenta en algunos actores que participan dentro y fuera de la burocracia estatal y que, como se mostró en este trabajo, son centrales, tal como la figura del coordinador barrial. A su vez, en su interés por ponderar la horizontalidad de las relaciones, algunos actores suelen pasar por alto las dinámicas (disputas, intereses) de poder que se presentan en entramados vinculados a la implementación de programas.

Sin embargo, debe señalarse que tampoco resulta suficiente una visión encerrada en la perspectiva clientelar de tipo instrumental. Tal como se ha mostrado en este trabajo, a pesar de que la red por la que se implementó el programa se conformó, en este caso de estudio, en contexto electoral, y de que los actores permanecen vinculados al partido gober- 
nante, no es posible afirmar que dichos actores estuviesen interesados principalmente en la acumulación de capital político con vistas a un contexto electoral. Como se ha mostrado, las relaciones sociales establecidas tenían sentido al lograr implementar el programa en cuestión. En afán de que los resultados del presente estudio se discutan con parte de la bibliografía sobre el tema en Argentina, y tal como vienen mostrando distintos autores, es necesario decir que las denominadas relaciones clientelares, entendidas de un modo instrumental, no pueden dar cuenta del conjunto de motivaciones, prácticas y relaciones sociales de los actores que implementan los programas.

También, se concluye a partir del caso que a través de las redes políticas preconstituidas la burocracia nacional logra funcionar: este tipo de redes ayuda a los funcionarios a seleccionar, tal como vimos en el presente caso, a los destinatarios de los programas y a conformar las cooperativas, a realizar cambios y adaptaciones rápidas sobre nuevos lineamientos enviados por los ministerios nacionales, y en general, a tener respuestas administrativas veloces.

Puede decirse también que las buenas intenciones vinculadas a las propuestas de fomentar el espíritu cooperativista y de economía social que están presentes en los lineamientos programáticos no son necesariamente retomadas en forma prioritaria por parte de los agentes que implementan los programas en el nivel municipal, pues estos tienden a reproducir lógicas y esquemas de programas sociales anteriores antes que involucrarse en la nueva propuesta ministerial.

A diferencia de la bibliografía sobre políticas públicas, la distancia establecida entre la formulación de políticas y su implementación no es interpretada en el presente trabajo como un fracaso del programa. Por el contrario, es dable pensar que hay un desencuentro entre las relaciones y prácticas históricamente arraigadas entre los actores sociales del nivel municipal y la planificación del programa en el nivel 
nacional. Esto permite pensar tanto en las diferentes modalidades bajo las que los programas nacionales pueden cobrar vida en territorios específicos como en la centralidad de los contextos para analizar la implementación de políticas.

Bibliografía

Aguilar Villanueva, L. F. (1994). La implementación de las politicas. México: Porrúa.

Alonso, G. (20I0). "La política social municipal y el problema de la coordinación interjurisdiccional. El caso del municipio bonaerense de General San Martín". Revista iberoamericana de estudios municipales, I (I), I7-45.

Alonso, G., y Di Costa, V. (2015). “Más allá del principio contributivo. Cambios y continuidades de la política social Argentina, 2003-20 I5”. Estudios sociológicos, 33(97), $3 \mid-62$.

Auyero, J. (1997). “Evita como performance. Mediación y resolución de problemas entre los pobres urbanos del Gran Buenos Aires", en J.Auyero (Comp.), ¿Favores por votos? Estudios sobre clientelismo político contemporáneo (pp. 167-232). Buenos Aires: Losada.

Auyero, J. (200I). La política de los pobres. Las prácticas clientelistas del peronismo. Buenos Aires: Manantial.

Bebbington, A. (2005). “Estrategias de vida y estrategias de intervención: el capital social y los programas de superación de la pobreza", en I. Arriagada (Ed.), Aprender de la experiencia. El capital social en la superación de la pobreza (PP. 2 I-46). Santiago de Chile: CEPAL.

Chaqués Bonafont, L. (2004). Redes de políticas públicas. Madrid: CIS.

Chiara, M., y Di Virgilio, M. M. (2005). Gestión social y municipios. De los escritorios del Banco Mundial a los barrios del Gran Buenos Aires. Buenos Aires: Prometeo, UNGS.

Cravacuore, D., llari, S., y Villar, A. (2004). La articulación en la gestión municipal. Actores y políticas. Buenos Aires: Editorial de la Universidad Nacional de Quilmes. 
Cravacuore, D., e Israel, R. (Comps.) (2007). Procesos políticos Bibliografía municipales comparados en Argentina y Chile (1990-2005). Buenos Aires: UNQ, Universidad Autónoma de Chile.

Di Natale, M. (2004). El festival de la pobreza. El uso político de planes sociales en Argentina. Buenos Aires: La Crujía Ediciones.

Durston, J. (2005). "Superación de la pobreza, capital social y clientelismos locales", en I. Arriagada (Ed.), Aprender de la experiencia. El capital social en la superación de la pobreza (pp. 47-58). Santiago de Chile: CEPAL.

Ilari, S. (2002a)."Fragmentación y articulaciones en la política social local", en D. Cravacuore (Comp.), Innovación en la gestión municipal (pp. 60-68). Buenos Aires: Universidad Nacional de Quilmes, Federación Argentina de Municipio. Ilari, S. (2002b). "La gestión local de la política social. Los casos de Quilmes y Florencio Varela”, en A. Ziccardi (Comp.), Pobreza, desigualdad social y ciudadanía: los límites de las políticas sociales en América Latina (pp. 377-402). Buenos Aires: CLACSO.

Isuani, F. (2009). “Redes intergubernamentales para la implementación de programas sociales”. RevistaAdministración pública y sociedad, (16), 37-62.

lucci, M. (2010). "Promoción de la inclusión social a través de programas sociales: los casos del Banco Popular de la Buena Fe y Argentina Trabaja". Revista de estudios regionales y mercado de trabajo, (6), 263-282.

lucci, M. (04 de abril de $20 \mathrm{I}$ la). Entrevista personal a Adrián, empleado administrativo de la Oficina de Cooperativas. Municipio de El Saladero, Buenos Aires, Argentina.

lucci, M. (05 de junio de 20 I lb). Entrevista personal a José, destinatario del programa en El Saladero. Buenos Aires, Argentina.

lucci, M. (03 de marzo de 20 I Ic). Entrevista personal a Pedro, director Oficina de Cooperativas del municipio de El Saladero. Buenos Aires, Argentina. 
Bibliografía

lucci, M. (05 de marzo de 20 I Id). Entrevista personal a Juan, vicedirector Oficina de Cooperativas del municipio de El Saladero. Buenos Aires, Argentina.

lucci, M. (03 de julio de 20 I le). Entrevista personal a Ramón, coordinador barrial del programa Argentina Trabaja en El Saladero. Buenos Aires, Argentina.

lucci, M. (27 de febrero de 20 I If). Entrevista personal a Norma, secretaria de Promoción Social del municipio de El Saladero. Buenos Aires, Argentina.

Jordana, J. (1995). "El análisis de los policy networks, una perspectiva sobre la relación entre políticas públicas y Estado". Revista Gestión y análisis de política pública, (3), 77-89.

Klijn, E. (2005). "Las redes de política pública. Una visión de conjunto”, en A. Cerrillo (Coord.), La gobernanza hoy: diez textos de referencia (pp. 2 I 3-244). Madrid: INAP. Levitsky, S. (2005). La transformación del justicialismo. Del partido sindical al partido clientelista, 1983-1999. Buenos Aires: Siglo XXI.

Lipsky, M. (1980). Street-level Bureaucracy Dilemmas of the Individual in Public Services. Nueva York: Russell Sage Foundation.

Ministerio de Desarrollo Social de la Nación (06 de agosto de 2009). Resolución ministerial $n^{\circ} 3 / 82$. Buenos Aires: Ministerio de Desarrollo Social, Presidencia de la Nación.

Ministerio de Desarrollo Social de la Nación (2010). ArgentinaTrabaja. Programa Ingreso Social con Trabajo. Guía informativa. Buenos Aires: Ministerio de Desarrollo Social, Presidencia de la Nación.

Ministerio de Desarrollo Social de la Nación (20I I). Programa Ingreso Social conTrabajo. Ejecución del programa. Principales resultados al primer semestre. Argentina: Ministerio de Desarrollo Social de la Nación, Presidencia de la Nación. 
Ortiz de Rozas, V. (2017). "Personal, particular y político: la producción del bien público en la práctica política cotidiana". Revista de sociologia e política, 25(63), 53-77.

Oszlack, O., y O’Donnell, G. (1995). "Estado y políticas estatales en América Latina: hacia una estrategia de investigación". Redes. Revista de estudios sociales de la ciencia, (4), 99-128.

Pressman, J., yWildavsky,A. (1998). Implementación. Cómo las grandes expectativas concebidas en Washington se frustran en Aokland. México: FCE.

Repetto, F. (2005). “La dimensión política de la coordinación de programas y políticas sociales: una aproximación teórica y algunas referencias prácticas en América Latina" en F. Repetto (Comp.), La gerencia social ante los nuevos retos del desarrollo social en América Latina (pp. 39-98). Guatemala: INAP-INDES-BID.

Soprano, G. (2008). “Doña Silvia. Análisis de redes políticas en el peronismo de la provincia de Misiones durante una campaña electoral municipal”. Andes, (19), I I9-I55.

Torres, J. P. (2002). Votos, chapas y fideos. Clientelismo político y ayuda social. La Plata: De la Campana.

Vommaro, G., y Quirós, J. (20I I).“Usted vino por su propia decisión: repensar el clientelismo en clave etnográfica”. Desacatos, (36), 65-84.

Yin, R. (20I3). Case study research:Design and methods. Nueva York: Sage.

Zarazaga, R. (20I5). "Los punteros como red de política social”. Postdata, 20(I), I I-29.

Zubriggen, C. (20II). "La utilidad del análisis de redes de políticas públicas”. Argumentos, 24(66), I8I-209. 